\title{
Evaluation of Spacing and Nipping Time under different Sowing Time in System of Chickpea Intensification for Climate Change Adaptation
}

\author{
H. L. Sonboir ${ }^{1 *}$, Vivek Tripathi ${ }^{1}$, L. K. Shrivastava ${ }^{2}$ and Sonendra Kumar ${ }^{1}$ \\ ${ }^{1}$ Department of Agronomy, India \\ ${ }^{2}$ Department of soil Science and Agricultural Chemistry, India \\ College of Agriculture, Indira Gandhi Krishi vishwavidyalaya, Raipur-492012, India \\ *Corresponding author
}

Keywords

Chickpea, Sowing

time, Nipping,

System of chickpea

intensification,

Nodulation, seed

yield, Economics,

Climate change

adaptation

Article Info

Accepted:

20 August 2019

Available Online:

10 September 2019

\section{A B S T R A C T}

A field experiment was laid out to evaluate effect of spacing and nipping time in different sowing time of chickpea at Agriculture Instructional cum Research Farm, IGKV, Raipur, Chhattisgarh during two consecutive winter seasons of 2016-17 and 2017-18 with four sowing time (First week of November, Third week of November, First week of December and Third week of December), three spacing (30x20, 40x20 and 50x20 cm) and three nipping time (No nipping, Nipping at 30 days, Nipping at 40 days). Nodulation and yield attributes were observed higher in November sowing with $50 \times 20 \mathrm{~cm}$ and $40 \times 20 \mathrm{~cm}$ spacing. Spacing of $50 \times 20 \mathrm{~cm}$ recorded higher seed yield (11.0 to $11.5 \%$ ) and net return (24.2 to $24.7 \%$ ) in first and third week of November sowing, moreover, spacing of $30 \times 20 \mathrm{~cm}$ recorded higher seed yield (11.0 to $12.6 \%$ ) and net return $(21.9$ to $23.1 \%)$ in third week of December sowing. Nipping at 30-40 DAS recorded increase of 4.8 to $7.5 \%$ in seed yield and 3.8 to $8.3 \%$ in net return in November sowing, however, it caused decrease of 9.3 to $12.2 \%$ in seed yield and 30.7 to 37.5 $\%$ in net return in third week of December sowing.

\section{Introduction}

Pulses are the most important source of protein in vegetarian diets. Looking into dietary essentiality of pulses, United nations declared 2016 as the International Year of Pulses. Chickpea is an important winter season pulse crop in India with 8.40 million hectare area and total production of 7.06 million metric tonnes during 2015-16 (Annual report, 2016-17). Chickpea is the most important crop grown during winter season in Chhattisgarh plain agro-climatic zone of India. It occupies 0.37 million ha with productivity of $1100 \mathrm{~kg} / \mathrm{ha}$ (Krishi Darshika, 2018). The low productivity of chickpea is mainly due to 
change in climatic condition, enhanced pest attack and improper agro-techniques particularly under climate change scenario.

As per projection, India will begin to experience more seasonal variation in temperature with more warming in the winter season (Kumar and Gautam, 2014) and an increase of average temperature of $1{ }^{\circ} \mathrm{C}$ can decrease yield of major food crops by 3.17.4\% (Zhao et al., 2017). Due to change in climatic condition winter season, chickpea is not performing well under the current recommended package of practices and shorter winter period exposes the chickpea crop often to hot condition during pod formation and grain filling stage. Hatfield and Prueger (2015) reported that warm temperature increased the rate of senescence during grain filling stage and reduced final grain yield.

There are several reports suggesting adaptation strategies to reduce ill effect of climate change such as, adjustment of planting dates, variety, crop relocation, improved land management, etc.

System of chickpea intensification has been reported to produce higher seed yield of chickpea compared to conventional sowing method recently (Sonboir and Tripathi, 2018, 2019) which is attributed with wider spacing and nipping practice. However, spacing and nipping practice can affect the chickpea crop in differently in varying sowing time as in changed climatic condition, the late sown crops has lesser biomass due to short winter period and exposure to heat during terminal stages. Therefore, a study was undertaken to know the effect of spacing and nipping time under varying sowing time in nodulation, yield and economic feasibility of chickpea under irrigated condition and to develop climate change adaptation strategies for chickpea.

\section{Materials and Methods}

A field experiment was conducted at Agriculture Instructional cum Research farm, IGK Raipur during two consecutive winter seasons of 2016-17 and 2017-18. The soil was clay in texture (Vertisols), neutral in soil reaction ( $\mathrm{pH} 7.1)$, normal in electrical conductivity $(0.16 \mathrm{dS} / \mathrm{m})$, low in available nitrogen $(219.7 \mathrm{~kg} / \mathrm{ha})$, medium in available phosphorus $(13.9 \mathrm{~kg} / \mathrm{ha})$ and high in potassium $(365.1 \mathrm{~kg} / \mathrm{ha})$. The experiment was laid out in split-split plot design with three replications. Main plot treatment consisted of four sowing time, viz. First week of November, third week of November, first week of December and third week of December, sub-plot treatments had three levels of spacing, viz. $30 \times 20 \mathrm{~cm}, 40 \times 20 \mathrm{~cm}$ and 50x20 cm and sub-sub plot treatments had three nipping time viz. No nipping, nipping at 30 days and nipping at 40 days. The test variety was JG 130. The chickpea crop was fertilized with 20:50:30 $\mathrm{kg} \mathrm{NP} \mathrm{O}_{5}: \mathrm{K}_{2} \mathrm{O} /$ ha at basal. The irrigation was given thrice, first just after sowing to ensure germination with $5 \mathrm{~cm}$ irrigation water and subsequent irrigation at 30 days and 50 days after sowing with $4 \mathrm{~cm}$ of irrigation water. The field were kept weed free through mechanical weeding at 20 and 40 days. The crop received 0.8 and $16.4 \mathrm{~mm}$ of rains during 2016-17 and 2017-18, respectively. During 2016-17, the winter season maximum temperature rising from $1^{\text {st }}$ week of February. Moreover, during 2017-18, maximum temperature started rising from $3^{\text {rd }}$ week of February. The number and dry weight of nodules were recorded at pre-flowering stage by destructive plant sampling with proper care. The seed and straw yield were recorded from net plot area after removing the border area. The gross return, cost of cultivation and net return were calculated from market price of the different inputs and outputs. B:C ratio was calculated by dividing gross return to cost of cultivation. All the data 
were subjected to analysis of variance as suggested by Gomez and Gomez (1983) for proper inference of results.

\section{Results and Discussion}

\section{Number and dry weight of nodules/plant}

Number and dry weight of nodules were significantly influenced by sowing time, spacing and nipping time (Table 1). Third week of November sowing exhibited maximum number of nodules per plant and nodule dry weight, which was however statistically similar to first week of November sowing. December sown chickpea crop showed decrease in nodules and nodule dry weight/plant. The lowest number and dry weight of nodules were recorded in third week of December sowing with significant difference to others. Similar report is also observed by Thalji and Shalaldeh (2006). Regarding spacing, 50x20 cm recorded more number and dry weight of nodules per plant which was however statistically at par with spacing of $40 \times 20 \mathrm{~cm}$. Closer spacing of 30x20 $\mathrm{cm}$ exhibited the lowest number and dry weight of nodules. Nipping time did not exert any significant difference in number and dry weight of nodules per plant.

\section{Yield attributes}

Number of pods/plant, number of seeds/pod and seed index were significantly influenced by sowing time, spacing and nipping time. Among all these yield attributes, only number of pods/plant differed significantly due to differences in sowing time.

Third week of November sowing exhibited significantly higher number of pods/plant, number of seeds/pod and seed index, statistically at par with first week of November sowing (Table 2). December sown chickpea crop showed decrease in number of pods/plant, seeds/pod and seed index. The lowest number of pods/plant, number of seeds/pod and seed index were recorded in third week of December sowing with significant difference to others. Spacing of $50 \times 20 \mathrm{~cm}$ spacing produced more number of pods/plant in all the sowing time with significant difference; however, it was par with $40 \times 20 \mathrm{~cm}$ spacing in first week of December sowing. Wider spacing of 50x20 $\mathrm{cm}$ spacing produced 29.8 to $31.7 \%$ more number of pods /plant as compared to closer spacing of $30 \times 20 \mathrm{~cm}$ in November sowing of chickpea. Increase in number of pods/plant with increase in row spacing is also reported by Sonboir et al., (2017). Third week of December sowing did not exert any significant difference in number of pods due to spacing (Table 3).

Nipping exerted significant contribution only in number of pods/plant in first and third week of November sowing and the maximum number of pods/plant was recorded with nipping at 40 days however it was at with nipping at 30 days.

Nipping recorded 15.3 to $21.2 \%$ increase in number of pods/plant in November sowing of chickpea. December first week sowing did not exhibited any significant difference in number of pods/plant while third week of December sowing recorded decrease in number of pods/plant due to nipping and the decrease was to the tune of 7.0 to $8.2 \%$. (Table 4 )

\section{Seed yield, straw yield and harvest index}

Seed yield, straw yield and harvest index were significantly influenced by sowing time, spacing and nipping time moreover, interaction effect of sowing time with spacing and nipping time were found significant. The variation in yield level was also observed in both years of study and the lower yield obtained during 2016-17 may be due to shorter winter period. 
First week of November sowing exhibited significantly higher seed yield, straw yield and harvest index, statistically at par with third week of November sowing (Table 5). December sown chickpea crop showed decrease in seed yield, straw yield and harvest index. The lowest seed yield, straw yield and harvest index were recorded in third week of December sowing with significant difference to others. The lower yield in late sown crop may be due heat stress during reproductive development (Devasirvatham et al., 2012). The maximum seed and straw was recorded with 50x20 cm spacing in first and third week of November sowing however it was statistically par with $40 \times 20 \mathrm{~cm}$ spacing in third week of November sowing. The average increase in seed yield was recorded 11.0 to $11.5 \%$ under $50 \times 20 \mathrm{~cm}$ spacing compared to closer spacing of $30 \times 20 \mathrm{~cm}$ in November sowing time. Higher yield under wider spacing in early sown crop may be due to better expression of growth parameters and availability of sufficient time to mature crop within desired temperature range. Similar result is also reported by Mondal (2000). Spacing of $40 \times 20 \mathrm{~cm}$ recorded maximum seed and straw yield in first week of December sowing time which was statistically at par with $30 \times 20 \mathrm{~cm}$ spacing. In third week of December sowing time, closer spacing of $30 \times 20 \mathrm{~cm}$ recorded maximum seed and straw yield with significant difference and the decreased the average seed yield to the tune of 11.0 to $12.6 \%$ due to increase in spacing (Table 6).

Nipping time exhibited significant effect on seed and straw yield in different sowing time (Table 7). The maximum seed yield was recorded in nipping at 40 days which was however statistically similar to nipping at 30 days in first and third week of November sowing time. Yield enhancement with nipping of terminal buds in chickpea is also reported by Baloch and Zubair (2010). The average increase in seed yield was 4.8 to $7.5 \%$ over no nipping in November sowing of chickpea. Nipping did not exhibit seed yield enhancement in December first week sowing time, however, higher straw yield was recorded under nipping at 30 days. No nipping recorded maximum seed and straw yield in third week of December sowing. Nipping caused 9.3 to $12.2 \%$ and decrease in seed yield over no nipping. The reduction in seed yield of chickpea with progressive delay in sowing beyond $10^{\text {th }}$ December is also reported by Shrivastava et al., (1990).

\section{Gross return, net return and B:C ratio}

Gross return, net return and $\mathrm{B}: \mathrm{C}$ ratio were significantly influenced by sowing time, spacing and nipping time moreover, interaction effect of sowing time with spacing and nipping time were found significant.

First week of November sowing recorded significantly higher gross return, net return and $\mathrm{B}: \mathrm{C}$ ratio followed by third week of November sowing (Table 8).

December sown chickpea crop showed more decrease in gross return, net return and B:C ratio. The lowest gross return, net return and $\mathrm{B}: \mathrm{C}$ ratio were recorded in third week of December sowing with significant difference.

$50 \times 20 \mathrm{~cm}$ spacing recorded higher gross return, net return and $\mathrm{B}: \mathrm{C}$ ratio in first and third week of November sowing. The average increase in net return was recorded 24.2 to $24.7 \%$ under $50 \times 20 \mathrm{~cm}$ spacing compared to closer spacing of $30 \times 20 \mathrm{~cm}$ in November sowing time. Spacing of $40 \times 20 \mathrm{~cm}$ recorded maximum gross, net return and $\mathrm{BC}$ ratio in first week of December sowing time which was statistically at par with $30 \times 20 \mathrm{~cm}$ spacing. Closer spacing of $30 \times 20 \mathrm{~cm}$ recorded maximum gross return, net return and $\mathrm{B}: \mathrm{C}$ ratio in third week of December sowing time with significant difference. 
The average decrease in net return was to the spacing in third week of December sowing tune of 21.9 to $23.1 \%$ due to increase in (Table 9).

Table.1 Number and dry weight of nodules at pre-flowering stage of chickpea as influenced by sowing time, spacing and nipping under SCI.

\begin{tabular}{|c|c|c|c|c|c|c|}
\hline \multirow[t]{2}{*}{ Treatments } & \multicolumn{3}{|c|}{ Number of nodules/plant } & \multicolumn{3}{|c|}{ Dry weight of nodules/plant, g } \\
\hline & 2016 & 2017 & Mean & 2016 & 2017 & Mean \\
\hline \multicolumn{7}{|l|}{ Sowing time } \\
\hline Nov. $1^{\text {st }}$ week & 34.3 & 57.4 & 45.9 & 0.57 & 0.78 & 0.68 \\
\hline Nov. $3^{\text {rd }}$ week & 45.9 & 48.3 & 47.1 & 0.72 & 0.69 & 0.71 \\
\hline Dec. $1^{\text {st }}$ week & 29.0 & 32.5 & 30.8 & 0.47 & 0.53 & 0.50 \\
\hline Dec. $3^{\text {rd }}$ week & 21.9 & 24.1 & 23.0 & 0.38 & 0.42 & 0.40 \\
\hline $\mathrm{CD}(\mathrm{p}=0.05)$ & 1.46 & 1.8 & 1.7 & 0.06 & 0.08 & 0.07 \\
\hline \multicolumn{7}{|l|}{ Spacing } \\
\hline $30 \times 20 \mathrm{~cm}$ & 31.8 & 39.5 & 35.7 & 0.51 & 0.75 & 0.63 \\
\hline $40 \times 20 \mathrm{~cm}$ & 32.6 & 40.7 & 36.7 & 0.53 & 0.79 & 0.66 \\
\hline $50 \times 20 \mathrm{~cm}$ & 34.0 & 41.6 & 37.8 & 0.56 & 0.82 & 0.69 \\
\hline $\mathrm{CD}(\mathrm{p}=0.05)$ & 1.4 & 1.6 & 1.5 & 0.02 & 0.03 & 0.03 \\
\hline \multicolumn{7}{|l|}{ Nipping time } \\
\hline No nipping & 32.0 & 40.3 & 36.2 & 0.52 & 0.75 & 0.64 \\
\hline Nipping at 30 DAS & 32.8 & 40.6 & 36.7 & 0.53 & 0.77 & 0.65 \\
\hline Nipping at 40 DAS & 33.5 & 40.8 & 37.2 & 0.55 & 0.78 & 0.67 \\
\hline $\mathrm{CD}(\mathrm{p}=0.05)$ & 1.2 & NS & NS & 0.02 & NS & NS \\
\hline
\end{tabular}

Table.2 Yield attributes of chickpea as influenced by sowing time, spacing and nipping under SCI.

\begin{tabular}{|c|c|c|c|c|c|c|c|c|c|}
\hline \multirow[t]{2}{*}{ Treatments } & \multicolumn{3}{|c|}{$\begin{array}{l}\text { Number of } \\
\text { pods/plant }\end{array}$} & \multicolumn{3}{|c|}{ Number of seeds/pod } & \multicolumn{3}{|c|}{ Seed index, $g$} \\
\hline & 2016 & 2017 & Mean & 2016 & 2017 & Mean & 2016 & 2017 & Mean \\
\hline \multicolumn{10}{|l|}{ Sowing time } \\
\hline Nov. $1^{\text {st }}$ week & 76.3 & 91.7 & 84.0 & 1.73 & 1.76 & 1.75 & 22.35 & 28.45 & 25.40 \\
\hline Nov. $3^{\text {rd }}$ week & 83.5 & 85.4 & 84.4 & 1.75 & 1.73 & 1.74 & 24.01 & 27.64 & 25.83 \\
\hline Dec. $1^{\text {st }}$ week & 57.0 & 60.2 & 58.6 & 1.62 & 1.64 & 1.63 & 20.25 & 26.52 & 23.39 \\
\hline Dec.3 ${ }^{\text {rd }}$ week & 44.5 & 47.9 & 46.2 & 1.48 & 1.52 & 1.50 & 18.47 & 24.53 & 21.50 \\
\hline $\mathrm{CD}(\mathrm{p}=0.05)$ & 2.4 & 4.6 & 3.5 & 0.04 & 0.06 & 0.05 & 0.63 & 0.84 & 0.74 \\
\hline \multicolumn{10}{|l|}{ Spacing } \\
\hline $30 \times 20 \mathrm{~cm}$ & 56.7 & 66.3 & 61.5 & 1.62 & 1.64 & 0.05 & 20.96 & 26.54 & 23.75 \\
\hline $40 \times 20 \mathrm{~cm}$ & 65.1 & 72.1 & 68.6 & 1.65 & 1.66 & 1.63 & 21.40 & 26.76 & 24.08 \\
\hline $50 \times 20 \mathrm{~cm}$ & 74.1 & 75.4 & 74.8 & 1.66 & 1.67 & 1.66 & 21.45 & 27.06 & 24.26 \\
\hline $\mathrm{CD}(\mathrm{p}=\mathbf{0 . 0 5})$ & 2.0 & 1.8 & 1.9 & NS & NS & NS & 0.32 & 0.42 & 0.37 \\
\hline \multicolumn{10}{|l|}{ Nipping time } \\
\hline No nipping & 62.5 & 68.2 & 65.4 & 1.62 & 1.65 & 1.64 & 20.98 & 26.75 & 23.87 \\
\hline Nipping at 30 DAS & 66.7 & 72.5 & 69.6 & 1.64 & 1.66 & 1.65 & 21.32 & 26.87 & 24.10 \\
\hline Nipping at 40 DAS & 66.8 & 73.3 & 70.0 & 1.67 & 1.66 & 1.67 & 21.50 & 26.76 & 24.13 \\
\hline $\mathrm{CD}(\mathrm{p}=0.05)$ & 1.6 & 1.5 & 1.6 & 0.04 & NS & NS & 0.30 & NS & NS \\
\hline
\end{tabular}


Table.3 Number of pods/plant of chickpea as influenced by interaction effect of sowing time and spacing under SCI.

\begin{tabular}{|c|c|c|c|c|c|c|c|c|c|}
\hline \multirow[t]{3}{*}{ Treatments } & \multicolumn{9}{|c|}{ Number of pods/plant } \\
\hline & \multicolumn{3}{|c|}{$30 \times 20 \mathrm{~cm}$} & \multicolumn{3}{|c|}{$40 \times 20 \mathrm{~cm}$} & \multicolumn{3}{|c|}{$50 \times 20 \mathrm{~cm}$} \\
\hline & 2016 & 2017 & Mean & 2016 & 2017 & Mean & 2016 & 2017 & Mean \\
\hline Nov. $1^{\text {st }}$ week & 64.0 & 80.5 & 72.3 & 74.0 & 95.1 & 84.6 & 90.9 & 99.5 & 95.2 \\
\hline Nov. $3^{\text {rd }}$ week & 68.2 & 78.7 & 73.5 & 83.6 & 85.3 & 84.5 & 98.6 & 92.1 & 95.4 \\
\hline Dec. $1^{\text {st }}$ week & 52.68 & 58.3 & 55.5 & 58.0 & 59.9 & 59.0 & 60.4 & 62.3 & 61.4 \\
\hline Dec. $3^{\text {rd }}$ week & 41.71 & 47.5 & 44.6 & 45.0 & 47.9 & 46.5 & 46.8 & 48.2 & 47.5 \\
\hline \multirow[t]{2}{*}{$\mathrm{CD}(\mathrm{p}=0.05)$} & \multicolumn{6}{|c|}{ Spacing at same sowing time } & 4.1 & 5.4 & 4.8 \\
\hline & \multicolumn{6}{|c|}{ Sowing time at same spacing } & 4.1 & 5.1 & 4.6 \\
\hline
\end{tabular}

Table.4 Number of pods/plant of chickpea as influenced by interaction effect of sowing time and nipping under SCI.

\begin{tabular}{|c|c|c|c|c|c|c|c|c|c|}
\hline \multirow[t]{3}{*}{ Treatments } & \multicolumn{9}{|c|}{ Number of pods/plant } \\
\hline & \multicolumn{3}{|c|}{ No nipping } & \multicolumn{3}{|c|}{ Nipping at 30 DAS } & \multicolumn{3}{|c|}{ Nipping at 40 DAS } \\
\hline & 2016 & 2017 & Mean & 2016 & 2017 & Mean & 2016 & 2017 & Mean \\
\hline Nov. $1^{\text {st }}$ week & 68.6 & 80.3 & 74.5 & 79.5 & 95.1 & 87.3 & 80.8 & 99.7 & 90.3 \\
\hline Nov. $3^{\text {rd }}$ week & 73.5 & 79.0 & 76.3 & 87.8 & 88.1 & 88.0 & 89.1 & 89.1 & 89.1 \\
\hline Dec. $1^{\text {st }}$ week & 60.5 & 63.4 & 62.0 & 56.1 & 59.8 & 58.0 & 54.5 & 57.4 & 56.0 \\
\hline Dec.3 $3^{\text {rd }}$ week & 47.4 & 50.0 & 48.7 & 43.4 & 47.1 & 45.3 & 42.7 & 46.6 & 44.7 \\
\hline \multirow[t]{2}{*}{$C D(p=0.05)$} & \multicolumn{6}{|c|}{ Nipping at same sowing time } & 3.2 & 4.8 & 4.0 \\
\hline & \multicolumn{6}{|c|}{ Sowing time at same nipping } & 3.5 & 4.3 & 3.9 \\
\hline
\end{tabular}

Table.5 Seed yield of chickpea as influenced by sowing time, spacing and nipping under SCI.

\begin{tabular}{|c|c|c|c|c|c|c|c|c|c|}
\hline \multirow[t]{2}{*}{ Treatments } & \multicolumn{3}{|c|}{ Seed yield, kg/ha } & \multicolumn{3}{|c|}{ Straw yield, kg/ha } & \multicolumn{3}{|c|}{ Harvest index } \\
\hline & 2016 & 2017 & Mean & 2016 & 2017 & Mean & 2016 & 2017 & Mean \\
\hline \multicolumn{10}{|l|}{ Sowing time } \\
\hline Nov. $1^{\text {st }}$ week & 2226 & 2889 & 2558 & 3897 & 4285 & 4091 & 36.33 & 40.27 & 38.30 \\
\hline Nov. $3^{\text {rd }}$ week & 2349 & 2573 & 2461 & 4118 & 3810 & 3964 & 36.31 & 40.31 & 38.31 \\
\hline Dec. $1^{\text {st }}$ week & 1827 & 2099 & 1963 & 3460 & 3318 & 3389 & 34.56 & 38.76 & 36.66 \\
\hline Dec. $3^{\text {rd }}$ week & 1411 & 1590 & 1501 & 2932 & 2871 & 2902 & 32.53 & 35.65 & 34.09 \\
\hline $\mathrm{CD}(\mathrm{p}=0.05)$ & 93 & 129 & 111 & 78 & 93 & 86 & 1.44 & 1.86 & 1.65 \\
\hline \multicolumn{10}{|l|}{ Spacing } \\
\hline $30 \times 20 \mathrm{~cm}$ & 1903 & 2276 & 2090 & 3605 & 3593 & 3599 & 34.43 & 38.78 & 36.61 \\
\hline $40 \times 20 \mathrm{~cm}$ & 1978 & 2296 & 2137 & 3604 & 3599 & 3602 & 35.16 & 38.94 & 37.05 \\
\hline $50 \times 20 \mathrm{~cm}$ & 1980 & 2292 & 2136 & 3596 & 3520 & 3558 & 35.20 & 39.43 & 37.32 \\
\hline $\mathrm{CD}(\mathrm{p}=0.05)$ & 51 & NS & NS & NS & NS & NS & NS & NS & NS \\
\hline \multicolumn{10}{|l|}{ Nipping time } \\
\hline No nipping & 1921 & 2271 & 2096 & 3539 & 3581 & 3560 & 35.03 & 38.81 & 36.92 \\
\hline Nipping at 30 DAS & 1993 & 2293 & 2143 & 3651 & 3557 & 3604 & 35.06 & 39.20 & 37.13 \\
\hline Nipping at 40 DAS & 1947 & 2299 & 2123 & 3614 & 3575 & 3595 & 34.71 & 39.15 & 36.93 \\
\hline $\mathrm{CD}(\mathrm{p}=0.05)$ & 30 & NS & NS & 52 & 58 & 55 & NS & 1.02 & NS \\
\hline
\end{tabular}


Table.6 Seed and straw yield of chickpea as influenced by interaction effect of sowing time and spacing under SCI.

\begin{tabular}{|c|c|c|c|c|c|c|c|c|c|}
\hline \multirow[t]{2}{*}{ Treatments } & \multicolumn{3}{|c|}{$30 \times 20 \mathrm{~cm}$} & \multicolumn{3}{|c|}{$40 \times 20 \mathrm{~cm}$} & \multicolumn{3}{|c|}{$50 \times 20 \mathrm{~cm}$} \\
\hline & 2016 & 2017 & Mean & 2016 & 2017 & Mean & 2016 & 2017 & Mean \\
\hline \multicolumn{10}{|c|}{ Seed yield, kg/ha } \\
\hline Nov. $1^{\text {st }}$ week & 2077 & 2787 & 2432 & 2260 & 2801 & 2531 & 2342 & 3079 & 2711 \\
\hline Nov. $3^{\text {rd }}$ week & 2159 & 2458 & 2309 & 2428 & 2595 & 2512 & 2460 & 2666 & 2563 \\
\hline Dec. $1^{\text {st }}$ week & 1834 & 2142 & 1988 & 1855 & 2258 & 2057 & 1794 & 1898 & 1846 \\
\hline Dec. $3^{\text {rd }}$ week & 1541 & 1718 & 1630 & 1370 & 1529 & 1450 & 1324 & 1524 & 1424 \\
\hline \multirow[t]{2}{*}{$C D(p=0.05)$} & \multicolumn{6}{|c|}{ Spacing at same sowing time } & 103 & 160 & 132 \\
\hline & \multicolumn{6}{|c|}{ Sowing time at same spacing } & 124 & 183 & 154 \\
\hline \multicolumn{10}{|c|}{ Straw yield, kg/ha } \\
\hline Nov. $1^{\text {st }}$ week & 3723 & 4186 & 3955 & 3909 & 4325 & 4117 & 4060 & 4344 & 4202 \\
\hline Nov. $3^{\text {rd }}$ week & 3973 & 3739 & 3856 & 4129 & 3766 & 3948 & 4251 & 3925 & 4088 \\
\hline Dec. $1^{\text {st }}$ week & 3462 & 3359 & 3411 & 3550 & 3509 & 3530 & 3366 & 3084 & 3225 \\
\hline Dec. $3^{\text {rd }}$ week & 3262 & 3087 & 3175 & 2827 & 2797 & 2812 & 2706 & 2728 & 2717 \\
\hline \multirow[t]{2}{*}{$C D(p=0.05)$} & \multicolumn{6}{|c|}{ Spacing at same sowing time } & 152 & 165 & 159 \\
\hline & \multicolumn{6}{|c|}{ Sowing time at same spacing } & 146 & 154 & 150 \\
\hline
\end{tabular}

Table.7 Seed and straw yield of chickpea as influenced by interaction effect of sowing time and nipping under SCI.

\begin{tabular}{|c|c|c|c|c|c|c|c|c|c|}
\hline \multirow[t]{2}{*}{ Treatments } & \multicolumn{3}{|c|}{ No nipping } & \multicolumn{3}{|c|}{ Nipping at 30 DAS } & \multicolumn{3}{|c|}{ Nipping at 40 DAS } \\
\hline & 2016 & 2017 & Mean & 2016 & 2017 & Mean & 2016 & 2017 & Mean \\
\hline \multicolumn{10}{|c|}{ Seed yield, kg/ha } \\
\hline Nov. $1^{\text {st }}$ week & 2119 & 2793 & 2456 & 2262 & 2909 & 2586 & 2299 & 2964 & 2632 \\
\hline Nov. $3^{\text {rd }}$ week & 2251 & 2476 & 2364 & 2387 & 2569 & 2478 & 2410 & 2673 & 2542 \\
\hline Dec. $1^{\text {st }}$ week & 1812 & 2081 & 1947 & 1937 & 2148 & 2043 & 1732 & 2069 & 1901 \\
\hline Dec. $3^{\text {rd }}$ week & 1501 & 1733 & 1617 & 1386 & 1546 & 1466 & 1347 & 1492 & 1420 \\
\hline \multirow[t]{2}{*}{$\mathrm{CD}(\mathrm{p}=0.05)$} & \multicolumn{6}{|c|}{ Nipping at same sowing time } & 60 & 120 & 90 \\
\hline & \multicolumn{6}{|c|}{ Sowing time at same nipping } & 104 & 161 & 133 \\
\hline \multicolumn{10}{|c|}{ Straw yield, kg/ha } \\
\hline Nov. $1^{\text {st }}$ week & 3742 & 4227 & 3985 & 3943 & 4278 & 4111 & 4007 & 4349 & 4178 \\
\hline Nov. $3^{\text {rd }}$ week & 3931 & 3769 & 3850 & 4190 & 3741 & 3966 & 4232 & 3920 & 4076 \\
\hline Dec. $1^{\text {st }}$ week & 3409 & 3302 & 3356 & 3570 & 3394 & 3482 & 3400 & 3257 & 3329 \\
\hline Dec. $3^{\text {rd }}$ week & 3539 & 3024 & 3282 & 3400 & 2815 & 3108 & 2818 & 2773 & 2796 \\
\hline \multirow[t]{2}{*}{$C D(p=0.05)$} & \multicolumn{6}{|c|}{ Nipping at same sowing time } & 104 & 134 & 119 \\
\hline & \multicolumn{6}{|c|}{ Sowing time at same nipping } & 115 & 119 & 117 \\
\hline
\end{tabular}


Table.8 Economics of chickpea as influenced by sowing time, spacing and nipping under SCI

\begin{tabular}{|c|c|c|c|c|c|c|c|c|c|}
\hline \multirow[t]{2}{*}{ Treatment } & \multicolumn{3}{|c|}{ Gross return, Rs/ha } & \multicolumn{3}{|c|}{ Net return, Rs/ha } & \multicolumn{3}{|c|}{ B:C ratio } \\
\hline & 2016 & 2017 & Mean & 2016 & 2017 & Mean & 2016 & 2017 & Mean \\
\hline \multicolumn{10}{|l|}{ Sowing time } \\
\hline Nov. $1^{\text {st }}$ week & 89059 & 124913 & 106986 & 52023 & 80500 & 66262 & 2.41 & 2.82 & 2.62 \\
\hline Nov. $3^{\text {rd }}$ week & 93972 & 111249 & 102611 & 56935 & 66836 & 61886 & 2.54 & 2.51 & 2.53 \\
\hline Dec. $1^{\text {st }}$ week & 73094 & 90887 & 81991 & 36057 & 46474 & 41266 & 1.98 & 2.05 & 2.02 \\
\hline Dec. $3^{\text {rd }}$ week & 56459 & 69028 & 62744 & 19422 & 24615 & 22019 & 1.53 & 1.56 & 1.55 \\
\hline$C D(p=0.05)$ & 3680 & 4765 & 4223 & 3680 & 4765 & 4223 & 0.10 & 0.13 & 0.12 \\
\hline \multicolumn{10}{|l|}{ Spacing } \\
\hline $30 \times 20 \mathrm{~cm}$ & 76107 & 98531 & 87319 & 37731 & 52532 & 45132 & 1.98 & 2.14 & 2.06 \\
\hline $40 \times 20 \mathrm{~cm}$ & 79128 & 99368 & 89248 & 42137 & 55010 & 48574 & 2.14 & 2.24 & 2.19 \\
\hline $50 \times 20 \mathrm{~cm}$ & 79203 & 99160 & 89182 & 43460 & 56278 & 49869 & 2.22 & 2.31 & 2.27 \\
\hline $\mathrm{CD}(\mathrm{p}=\mathbf{0 . 0 5})$ & 2058 & NS & 1045 & 2058 & NS & 1045 & 0.06 & 0.07 & 0.07 \\
\hline \multicolumn{10}{|l|}{ Nipping time } \\
\hline No nipping & 76834 & 98306 & 87570 & 41164 & 55766 & 48465 & 2.16 & 2.31 & 2.24 \\
\hline Nipping at 30 DAS & 79717 & 99238 & 89478 & 41997 & 53888 & 47943 & 2.12 & 2.19 & 2.16 \\
\hline Nipping at 40 DAS & 77888 & 99514 & 88701 & 40168 & 54165 & 47167 & 2.07 & 2.20 & 2.14 \\
\hline $\mathrm{CD}(\mathrm{p}=0.05)$ & 1193 & 1134 & 1164 & 1193 & 1134 & 1164 & 0.03 & 0.04 & 0.04 \\
\hline
\end{tabular}

Table.9 Economics of chickpea as influenced by interaction effect of sowing time and spacing under SCI

\begin{tabular}{|c|c|c|c|c|c|c|c|c|c|}
\hline \multirow[t]{2}{*}{ Treatments } & \multicolumn{3}{|c|}{$30 \times 20 \mathrm{~cm}$} & \multicolumn{3}{|c|}{$40 \times 20 \mathrm{~cm}$} & \multicolumn{3}{|c|}{$50 \times 20 \mathrm{~cm}$} \\
\hline & 2016 & 2017 & Mean & 2016 & 2017 & Mean & 2016 & 2017 & Mean \\
\hline \multicolumn{10}{|c|}{ Gross return, kg/ha } \\
\hline Nov. $1^{\text {st }}$ week & 83082 & 120527 & 101805 & 90406 & 121198 & 105802 & 93690 & 133014 & 113352 \\
\hline Nov. $3^{\text {rd }}$ week & 86377 & 106316 & 96347 & 97129 & 112158 & 104644 & 98411 & 115275 & 106843 \\
\hline Dec. $1^{\text {st }}$ week & 73349 & 92721 & 83035 & 74183 & 97733 & 85958 & 71749 & 82207 & 76978 \\
\hline Dec. $3^{\text {rd }}$ week & 61622 & 74558 & 68090 & 54792 & 66383 & 60588 & 52963 & 66143 & 59553 \\
\hline \multirow[t]{2}{*}{$\mathrm{CD}(\mathrm{p}=0.05)$} & \multicolumn{6}{|c|}{ Spacing at same sowing time } & 4115 & 5321 & 4718 \\
\hline & \multicolumn{6}{|c|}{ Sowing time at same spacing } & 4971 & 6124 & 5548 \\
\hline \multicolumn{10}{|c|}{ Net return, kg/ha } \\
\hline Nov. $1^{\text {st }}$ week & 44705 & 74528 & 59617 & 53416 & 76840 & 65128 & 57947 & 90132 & 74040 \\
\hline Nov. $3^{\text {rd }}$ week & 48000 & 60317 & 54159 & 60138 & 67799 & 63969 & 62668 & 72393 & 67531 \\
\hline Dec. $1^{\text {st }}$ week & 34972 & 46722 & 40847 & 37193 & 53375 & 45284 & 36006 & 39325 & 37666 \\
\hline Dec. $3^{\text {rd }}$ week & 23245 & 28560 & 25903 & 17801 & 22025 & 19913 & 17220 & 23261 & 20241 \\
\hline \multirow[t]{2}{*}{$\mathrm{CD}(\mathrm{p}=\mathbf{0 . 0 5})$} & \multicolumn{6}{|c|}{ Spacing at same sowing time } & 4115 & 5321 & 4718 \\
\hline & \multicolumn{6}{|c|}{ Sowing time at same spacing } & 4971 & 6124 & 5548 \\
\hline \multicolumn{10}{|c|}{ B:C ratio } \\
\hline Nov. $1^{\text {st }}$ week & 2.16 & 2.62 & 2.39 & 2.44 & 2.73 & 2.59 & 2.62 & 3.10 & 2.86 \\
\hline Nov. $3^{\text {rd }}$ week & 2.25 & 2.31 & 2.28 & 2.63 & 2.53 & 2.58 & 2.75 & 2.69 & 2.72 \\
\hline Dec. $1^{\text {st }}$ week & 1.91 & 2.02 & 1.97 & 2.01 & 2.20 & 2.11 & 2.01 & 1.92 & 1.97 \\
\hline Dec. $3^{\text {rd }}$ week & 1.61 & 1.63 & 1.62 & 1.48 & 1.50 & 1.49 & 1.49 & 1.55 & 1.52 \\
\hline \multirow[t]{2}{*}{$\mathrm{CD}(\mathrm{p}=\mathbf{0 . 0 5})$} & \multicolumn{6}{|c|}{ Spacing at same sowing time } & 0.11 & 0.14 & 0.13 \\
\hline & \multicolumn{6}{|c|}{ Sowing time at same spacing } & 0.14 & 0.19 & 0.17 \\
\hline
\end{tabular}


Table.10 Economics of chickpea as influenced by interaction effect of sowing time and nipping under SCI

\begin{tabular}{|c|c|c|c|c|c|c|c|c|c|}
\hline \multirow[t]{2}{*}{ Treatments } & \multicolumn{3}{|c|}{ No nipping } & \multicolumn{3}{|c|}{ Nipping at 30 DAS } & \multicolumn{3}{|c|}{ Nipping at 40 DAS } \\
\hline & 2016 & 2017 & Mean & 2016 & 2017 & Mean & 2016 & 2017 & Mean \\
\hline \multicolumn{10}{|c|}{ Gross return, Rs/ha } \\
\hline Nov. $1^{\text {st }}$ week & 84748 & 120836 & 102792 & 90475 & 125759 & 108117 & 91954 & 128145 & 110050 \\
\hline Nov. $3^{\text {rd }}$ week & 90041 & 107136 & 98589 & 95470 & 111072 & 103271 & 96406 & 115541 & 105974 \\
\hline Dec. $1^{\text {st }}$ week & 72492 & 90104 & 81298 & 77497 & 93001 & 85249 & 69293 & 89557 & 79425 \\
\hline Dec. $3^{\text {rd }}$ week & 60055 & 75150 & 67603 & 55424 & 67120 & 61272 & 53897 & 64815 & 59356 \\
\hline \multirow[t]{2}{*}{$C D(p=0.05)$} & \multicolumn{6}{|c|}{ Nipping at same sowing time } & 2385 & 3576 & 2981 \\
\hline & \multicolumn{6}{|c|}{ Sowing time at same nipping } & 4149 & 4895 & 4522 \\
\hline \multicolumn{10}{|c|}{ Net return, $\mathrm{Rs} / \mathrm{ha}$} \\
\hline Nov. $1^{\text {st }}$ week & 49078 & 78296 & 63687 & 52755 & 80409 & 66582 & 54234 & 82795 & 68515 \\
\hline Nov. $3^{\text {rd }}$ week & 54371 & 64596 & 59484 & 57750 & 65722 & 61736 & 58686 & 70191 & 64439 \\
\hline Dec. $1^{\text {st }}$ week & 36822 & 47564 & 42193 & 39777 & 47651 & 43714 & 31573 & 44207 & 37890 \\
\hline Dec. $3^{\text {rd }}$ week & 24385 & 32610 & 28498 & 17704 & 21771 & 19738 & 16177 & 19465 & 17821 \\
\hline \multirow[t]{2}{*}{$\mathrm{CD}(\mathrm{p}=0.05)$} & \multicolumn{6}{|c|}{ Nipping at same sowing time } & 2385 & 3576 & 2981 \\
\hline & \multicolumn{6}{|c|}{ Sowing time at same nipping } & 4149 & 4895 & 4522 \\
\hline \multicolumn{10}{|c|}{ B:C ratio } \\
\hline Nov. $1^{\text {st }}$ week & 2.38 & 2.85 & 2.62 & 2.40 & 2.78 & 2.59 & 2.44 & 2.83 & 2.64 \\
\hline Nov. $3^{\text {rd }}$ week & 2.53 & 2.52 & 2.53 & 2.54 & 2.45 & 2.50 & 2.56 & 2.55 & 2.56 \\
\hline Dec. $1^{\text {st }}$ week & 2.03 & 2.12 & 2.08 & 2.06 & 2.05 & 2.06 & 1.84 & 1.97 & 1.91 \\
\hline Dec. $3^{\text {rd }}$ week & 1.68 & 1.77 & 1.73 & 1.47 & 1.48 & 1.48 & 1.43 & 1.43 & 1.43 \\
\hline \multirow[t]{2}{*}{$\mathrm{CD}(\mathrm{p}=0.05)$} & \multicolumn{6}{|c|}{ Nipping at same sowing time } & 0.07 & 0.08 & 0.08 \\
\hline & \multicolumn{6}{|c|}{ Sowing time at same nipping } & 0.11 & 0.14 & 0.13 \\
\hline
\end{tabular}

Nipping time exhibited significant effect on gross return, net return and $\mathrm{B}$ : ratio in different sowing time (Table 10). The maximum gross return and net return was recorded in nipping at 40 days which was however statistically similar to nipping at 30 days in first and third week of November sowing time.

The average increase in net return was 3.8 to $8.3 \%$ over no nipping in November sowing of chickpea. Nipping at 30 days recorded maximum gross return and net return in first week of November sowing, moreover, net return was at par with no nipping in first week of November sowing. No nipping recorded maximum gross return and net return in third week of December sowing. Nipping caused
30.7 to $37.5 \%$ decrease in net return over no nipping. B: ratio was recorded higher in first and third week of November sowing. Spacing and nipping time exerted significant difference in different sowing time. The maximum yield attributes, seed yield, straw yield, gross return and net return were recorded with $50 \times 20 \mathrm{~cm}$ spacing and nipping at 40 days which was however statistically similar to nipping at 30 days in first and third week of November sowing. No nipping recorded maximum seed yield, straw yield, gross return and net return in third week of December sowing. Thus, in changing climate scenario, wider spacing with nipping operation at 30-40 days for higher yield and net return in early sown crop in the month of November and closer spacing of $30 \times 20 \mathrm{~cm}$ spacing without nipping operation 
in delayed sowing of third week of December can be practiced to minimize the losses in seed yield and net return of chickpea.

\section{References}

Annual report 2016-17. Department of Agriculture, Cooperation and Farmers welfare, Directorate of pulse development Vindhyachal Bhavan, Govt of India, p 9

Baloch, M. S. and Zubair, M. 2010. Effect of nipping on growth and yield of chickpea. The Journal of Animal \& Plant Sciences, 20(3): 208-210

Devasirvatham, V., Tan, D. K. Y., Gaur, P. M., Raju, T. N., Trethowan, R. M. 2012. High temperature tolerance in chickpea and its implications for plant improvement. Crop and Pasture Science, 63(5):419-428

Gomez, K. A. and Gomez, A. A. 1983. Statistical procedures for agricultural research. An International Rice Research Institute book, John Wiley and Sons, New York.

Hatfield, J. L. and Prueger, J. H. 2015. Temperature extremes: effect on plant growth and development. Weather and climate extremes, 10 (A) page 4-10.

Krishi darshika, 2018. Area and productivity of different crops in Chhattisgarh. Krishi Darshika Published by Indira Gandhi Krishi Vishwavidyalaya, Raipur PP 6.

Kumar, R. and Gautam, H. R. 2014. Climate Change and its Impact on Agricultural Productivity in India Journal of Climatology \& Weather Forecasting 2 (1): 1000109.

Mondal, S. 2000. Response of chickpea varieties (Cicer arietinum L.) to dats of sowing and row spacing under late sown condition. M.Sc. (Agronomy) thesis submitted to G.B. Pant University of Agriculture and Technology, Pantanagar, India.

Shrivastava, S. K., Singh, R. and Chandrawanshi, B. R. 1990. Response of chickpea cultivars under different dates of sowing in Chhattisgarh region of Madhya Pradesh. International Chickpea news letter, 23: 26-27.

Sonboir, H. L., Sahu, B.K. and Vivek Tripathi. 2017. Evaluation of row spacing and nipping on productivity and profitability of chickpea under irrigated condition. Green Farming 8(2): 422-425.

Sonboir, H. L. and Vivek Tripathi. 2018. System of chickpea intensification-A new initiative to enhance productivity of irrigated chickpea. Newsletter, India society of Agronomy, ICAR-IARI, New Delhi, April to September Vol 2 (2\&3):5-6

Sonboir, H. L. and Vivek Tripathi. 2019. An innovative approach to enhance chickpea productivity under irrigated condition-system of chickpea intensification. Institutional innovations and interventions, Management practices for crops and allied enterprises published by Indira Gandhi Agriculture University technical cell, Volume I: 1-5

Thalji, T. and Shalaldeh, G. 2006. Effect of planting date on Faba bean (Vicia faba L.) nodulation and performance under semiarid conditions. World Journal of Agricultural Sciences, 2(4): 477-482.

Zhao, C., Liu, B., Piao, S. and Wang, H. 2017. Temperature increase reduces global yields of major crops in four independent estimates. In Proceeding of the national academy of sciences 114 (35) 9326-9331. 


\section{How to cite this article:}

Sonboir H. L., Vivek Tripathi, L. K. Shrivastava and Sonendra Kumar 2019. Evaluation of Spacing and Nipping Time under different Sowing Time in System of Chickpea Intensification for Climate Change Adaptation. Int.J.Curr.Microbiol.App.Sci. 8(09): 1858-1868 doi: https://doi.org/10.20546/ijcmas.2019.809.215 